\title{
Burkholderia cepacia-Induced IL-8 Gene Expression in an Alveolar Epithelial Cell Line: Signaling Through CD14 and Mitogen-Activated Protein Kinase
}

\author{
KRISANAVANE REDDI, STEPHEN B. PHAGOO, KATHRYN D. ANDERSON, AND \\ DAVID WARBURTON
}

\begin{abstract}
Developmental Biology Program [K.R., S.B.P., D.W.] and Department of Pediatric Surgery [K.D.A., D.W.], Childrens Hospital, Los Angeles Research Institute (CHLARI), Los Angeles, California 90027, U.S.A.
\end{abstract}

\begin{abstract}
Burkholderia cepacia is a prevalent pulmonary pathogen in patients with cystic fibrosis (CF). The lung pathology observed in patients with $\mathrm{CF}$ is postulated to be due to an overexpression of chemokines. This study investigated the induction of the neutrophil chemoattractant chemokine IL-8 and the signaling pathways activated by $B$. cepacia-infected human lung epithelial A549 (HLE) cells. Cells were infected with B. cepacia (genomovar III of the $B$. cepacia complex), and reverse transcriptase-PCR and ELISA for the cytokines were performed. B. cepacia (multiplicity of infection $\geq 4: 1$ ) induced HLE cells to significantly secrete IL-8 in a more potent manner than the predominant CF pathogen Pseudomonas aeruginosa (multiplicity of infection $\geq 64: 1$ ). IL-8 secretion by $B$. cepacia-infected HLE cells was abrogated by the gene transcription inhibitor actinomycin $\mathrm{D}$ and the protein translation inhibitor cycloheximide, confirming that B. cepaciainduced IL-8 secretion was mediated through de novo protein synthesis. Treatment of $B$. cepacia with proteinase $\mathrm{K}$ failed to down-regulate IL-8 secretion; furthermore, IL-8 secretion by $B$. cepacia-infected HLE cells was abrogated by $\geq 80 \%$ in the presence of anti-CD14 [specific lipopolysaccharide (LPS) receptor] antibody, thus suggesting that the IL-8-inducing component of $B$. cepacia was LPS and therefore dependent on CD14. The p38 mitogen-activated protein kinase (MAPK) inhibitor and the
\end{abstract}

\section{ABSTRACT}

extracellular signal-regulated kinase MAPK inhibitor significantly abrogated IL-8 secretion by $B$. cepacia-infected HLE cells (SB203580, $\geq 80 \%$ inhibition; PD98059, $\geq 30 \%$ inhibition). In conclusion, B. cepacia-induced IL-8 secretion in A549 airway epithelial cells is more potent than $P$. aeruginosa; is mediated through LPS, which is CD14 dependent; and involves activation of the p38 and ERK MAPK pathways. (Pediatr Res 54: 297305, 2003)

$\quad$ Abbreviations
$\boldsymbol{\alpha}$ CD14, anti-CD14 receptor antibody
CF, cystic fibrosis
ERK, extracellular signal-regulated kinase
HLE, human lung epithelial
JNK, c-Jun $\mathrm{NH}_{2}$-terminal kinase
LBP, lipopolysaccharide-binding protein
LPS, lipopolysaccharide
MAPK, mitogen activated protein kinase
MOI, multiplicity of infection
PK, proteinase K
PKC, protein kinase C
PMSF, phenylmethylsulfonyl fluoride
TNF- $\alpha$, tumor necrosis factor- $\alpha$

Cystic fibrosis (CF) is the most common lethal genetic disease of white individuals (1). Exaggerated and persistent

Received January 16, 2002; accepted October 30, 2002.

Correspondence: David Warburton, D.Sc., M.D., Developmental Biology Program, Childrens Hospital Los Angeles Research Institute, 4650 Sunset Boulevard, MS\#35, Los Angeles, CA 90027, U.S.A.; e-mail: dwarburton@chla.usc.edu

Supported by National Institutes of Health Program Project Grant HL60231, the Cystic Fibrosis Foundation (S.B.P.), and a fellowship from the CHLARI (S.B.P.).

DOI: 10.1203/01.PDR.0000076661.85928.1D chronic inflammation caused by microbial colonization of the major airways is thought to be one of the major causes of morbidity and mortality in patients with CF (2). One of the major organisms to infect patients with CF is Pseudomonas aeruginosa. However, in recent years, the multidrug-resistant pulmonary pathogen Burkholderia cepacia has become increasingly prevalent in these patients (3). Approximately $20 \%$ of $B$. cepacia-infected patients eventually experience " $B$. ce- 
pacia syndrome." The symptoms of this syndrome include bacteremia and leukocytosis that result in rapid and fatal clinical deterioration (4) associated with increased levels of cytokines in the blood and sputa $(5,6)$.

Several studies have reported a marked increase in proinflammatory cytokines in patients with $\mathrm{CF}$ (7), in particular IL-8. As IL-8 is a potent chemokine for the attraction of neutrophils to the lung, an excess of this chemokine leads to a large influx of neutrophils that ultimately contribute to the lung damage observed in patients with CF. The signaling pathways activated during bacterial induction of chemokine gene expression have not been fully elucidated. However, the mitogenactivated protein kinases (MAPKs) have been demonstrated to play a role in regulating intracellular signal transduction during mononuclear cell cytokine production in response to a variety of stimuli (8). Members of the MAPK family include extracellular signal-regulated kinase (ERK) MAPK, c-Jun $\mathrm{NH}_{2}-$ terminal kinases (JNKs), and p38 MAPK. Activation of p38 MAPK before tumor necrosis- $\alpha$ (TNF- $\alpha$ ) synthesis is known to occur in human neutrophils poststimulation with lipopolysaccharide (LPS) (9). However, the signaling pathways activated by $B$. cepacia to induce IL- 8 synthesis remain unclear.

Respiratory epithelial cells are one of the main cell types encountered by $B$. cepacia infecting the CF lung $(10,11)$. A recent report by Keig et al. (12) demonstrated that $B$. cepacia is capable of invading and surviving within primary type II pneumocytes, and they and others have concluded that the transformed lung epithelial cell line A549 can be used as a valid surrogate for type II pneumocytes $(10,12-15)$. Hence, in this study, we used A549 cells as a surrogate for type II pneumocytes to evaluate the IL-8-inducing ability of B. cepacia.

Several studies have suggested that $P$. aeruginosa is stimulatory for cytokine synthesis in epithelial cells. In this study, we provide novel evidence that a clinical isolate of $B$. cepacia (ATCC 700070, genomovar III of the $B$. cepacia complex) is more potent than $P$. aeruginosa at inducing IL-8 in airway epithelial cells. We also report that B. cepacia-induced IL-8 secretion in airway epithelial cells is CD14 dependent and involves the p38 and ERK MAPK pathway.

\section{METHODS}

Bacterial strains and culture conditions. B. cepacia was obtained from the American Tissue Culture Collection (ATCC) 700070, a clinical isolate from a patient with CF that has been reported to be resistant to chloramphenicol, trimethoprim, and ciprofloxacin (16). B. cepacia ATCC strain 700070 was confirmed as belonging to genomovar III by rDNA and recA gene analyses (J. Lipuma, personal communication). B. cepacia was routinely cultured on nutrient agar (Sigma Chemical Co., St. Louis, MO, U.S.A.) without any supplements and incubated at $37^{\circ} \mathrm{C}$ for $48 \mathrm{~h}$. The IL-8 inducing abilities of both $\mathrm{B}$. cepacia and the laboratory strain of $P$. aeruginosa PAO1 (ATCC) were compared. $P$. aeruginosa was cultured in a similar manner to $B$. cepacia as described above. One day before experiments, the organisms were cultured overnight in nutrient broth at $37^{\circ} \mathrm{C}$, and the number of organisms $/ \mathrm{mL}$ was calculated by standard optical density measurements. All optical density calculations were checked by performing direct counts of the organisms by the use of a Petroff-Hausser counting chamber (Hausser Scientific, Horshem, PA, U.S.A.).

Cell culture. Human lung epithelial (HLE) cells A549 (ATCC CCL185) were cultured in RPMI 1640 medium (Sigma Chemical Co.) containing 10\% heat-inactivated fetal bovine serum (HI-FBS) and $50 \mathrm{IU} / \mathrm{mL}$ of penicillin and $50 \mu \mathrm{g} / \mathrm{mL}$ of streptomycin. Before the cytokine assays, the cells were passaged once in the absence of antibiotics and used throughout the assay without antibiotics. For all experiments, the cells were plated at a cell density of 70,000 cells $/ \mathrm{mL}$ in their respective medium containing $2 \% \mathrm{HI}-\mathrm{FBS}$ and incubated for $18 \mathrm{~h}$ at $37^{\circ} \mathrm{C}$ in $5 \% \mathrm{CO}_{2} / 95 \%$ air before infecting with various multiplicity of infections (MOIs) of B. cepacia or P. aeruginosa. An MOI of 2:1 refers to two bacteria per one HLE cell.

$I L-\boldsymbol{\delta}$ and $\boldsymbol{I L - 1} \boldsymbol{\beta}$ assays. ELISA 24-well plates (Corning, New York, NY, U.S.A.) were cultured with HLE cells at the density mentioned above and incubated overnight (see above). The confluent monolayer was washed three times with PBS, and then either $B$. cepacia or $P$. aeruginosa was added at the MOI stated in the figure legends. Controls consisted of RPMI 1640 plus $2 \%$ HI-FBS as a negative control and IL-1 $\beta$ (500 $\mathrm{pg} / \mathrm{mL}$ ) as a positive control. The plates were incubated for $18 \mathrm{~h}$, and the viability of the cells was determined by trypan blue stain by standard methods. The cell medium was removed, centrifuged at $13,000 \times g$ for $15 \mathrm{~min}$ to remove bacteria and cell debris, and then stored at $-80^{\circ} \mathrm{C}$ until assayed for IL-8 and IL-1 $\beta$ by ELISA as described by Steffen et al. (17).

Protease treatment of B. cepacia. To determine whether a protein component of $B$. cepacia was responsible for IL-8 secretion in HLE cells, we treated B. cepacia (MOI 50:1) with increasing concentrations of proteinase $\mathrm{K}(\mathrm{PK} ; 0.1-0.5 \mathrm{mg} /$ $\mathrm{mL}$; Sigma Chemical Co.) and incubated it at $37^{\circ} \mathrm{C}$ for $1 \mathrm{~h}$ as described by Weinberg et al. (18). Control bacterial suspensions without the PK were incubated under identical conditions. The reaction was then terminated by the addition of phenylmethylsulfonyl fluoride (PMSF) and incubated for an additional $30 \mathrm{~min}$ at $37^{\circ} \mathrm{C} \mathrm{(18).} \mathrm{The} \mathrm{bacterial} \mathrm{suspensions} \mathrm{were}$ then washed, and the resulting bacterial pellets suspended in PBS to their original volumes. HLE cells were then exposed to either PK-treated or untreated B. cepacia (MOI 50:1) incubated for $18 \mathrm{~h}$ and IL- 8 secreted into the culture medium quantified by ELISA. Controls included PK at the highest concentration tested plus PMSF in the absence of B. cepacia and medium only. For confirming that PK treatment had completely digested the bacterial proteins, a sample of the PK-treated $B$. cepacia was run on a SDS-PAGE gel.

Reverse transcriptase-PCR. HLE cells were grown to confluence in six-well dishes (Falcon, NJ) and incubated in RPMI 1640 with $2 \%$ HI-FBS for $18 \mathrm{~h}$ in the presence of B. cepacia (MOI 50:1). Cell viability was checked at the conclusion of experiments by trypan blue exclusion. All cells had a viability of $>95 \%$. Total RNA was extracted from the cells using TRIZOL reagent as described by the manufacturer ( $\mathrm{GIBCO} /$ BRL, Carlsbad, NY, U.S.A.). Single-stranded cDNA was generated as described by Jung et al. (19). Amplification of cDNA by PCR was performed using oligonucleotide primer pairs for IL-8 or IL- $1 \beta$ as described by Jung et al. (19). $\beta$-Actin was 
used to control for loading density. The PCR reactions were carried out in a thermal cycler (Perkin-Elmer) in a $28-\mu \mathrm{L}$ reaction volume, containing reaction buffer $[20 \mathrm{mM}$ of Tris/ HCL (pH 8.4), $50 \mathrm{mM}$ of KCl], $1.5 \mathrm{mM}$ of $\mathrm{MgCl}_{2}, 0.2 \mathrm{mM}$ of dNTP mix (dATP, dCTP, dGTP, dTTP), $1.5 \mathrm{U}$ of Taq polymerase $(\mathrm{GIBCO} / \mathrm{BRL})$, and $2 \mu \mathrm{L}$ of first-strand reaction mix. Each primer was added to a final concentration of $0.1 \mu \mathrm{M}$. PCR was for 35 cycles, each cycle consisting of $1 \mathrm{~min}$ at $95^{\circ} \mathrm{C}$ and annealing/extension at $60^{\circ} \mathrm{C}$ for $2 \mathrm{~min}$ and $30 \mathrm{~s}$. The PCR products were separated on $1 \%$ agarose gels containing 50 $\mu \mathrm{g} / \mathrm{mL}$ of ethidium bromide, visualized, and photographed under UV light. Densitometry readings of the bands were performed using the Scion Image for Windows program.

Involvement of CD14 receptor. To assess whether CD14 receptors are involved during $B$. cepacia-mediated IL-8 synthesis in HLE cells, we preincubated these cells with either anti-CD14 antibody (MY4; Beckman Coulter, Brea, CA, U.S.A.) or an isotypic control antibody (IgG2b; Beckman Coulter) $1 \mathrm{~h}$ before infecting with B. cepacia (MOI 50:1) or LPS from $P$. aeruginosa (serotype 10,100 $\mu \mathrm{g} / \mathrm{mL}$; Sigma Chemical Co.). After incubating for $18 \mathrm{~h}$, the IL-8 secreted into the culture medium was quantified by ELISA. Controls consisted of either media alone or B. cepacia or P. aeruginosa LPS in the absence of antibodies.

Assessment of signaling proteins: ELISA and Western analysis. For determining whether the MAPK pathways are activated by HLE cells in response to B. cepacia to secrete IL-8, HLE cells were preincubated with either the p38 MAPK inhibitor SB203580 (1-3 $\mu \mathrm{M}$; Calbiochem, La Jolla, CA, U.S.A.) or the ERK MAPK inhibitor PD98059 (10 $\mu \mathrm{M}$; Calbiochem) for $1 \mathrm{~h}$. These cells were then exposed to $B$. cepacia for $18 \mathrm{~h}$, and the IL-8 secreted into the culture medium was quantified by ELISA. Controls consisted of either media only or B. cepacia in the absence of inhibitors. Western analysis was used to assess whether there was an increase in the protein for phosphorylated p38 MAPK postexposure to $B$. cepacia in HLE cells. Confluent HLE cells were cultured in six-well plates and exposed to $B$. cepacia (MOI 50:1) in the absence and presence of $3 \mu \mathrm{M}$ of SB203580 for $10 \mathrm{~min}$. The cells were then lysed with $200 \mu \mathrm{L}$ of RIPA lysis buffer (containing $60 \mu \mathrm{M}$ of PMSF, $30 \mathrm{U} / \mathrm{mL}$ of aprotinin, and $1 \mathrm{mM}$ of sodium orthovanadate; Sigma Chemical Co.). Before loading onto 10\% SDS polyacrylamide gels (Invitrogen, Carlsbad, CA, U.S.A.), the samples were treated with sample buffer and then denatured by boiling for $5 \mathrm{~min}$. Gels were run at $200 \mathrm{~mA}$ for $1.5 \mathrm{~h}$ at room temperature. Proteins were transferred onto Hybond-ECL nitrocellulose paper (Millipore, Bedford, MA, U.S.A.) in blotting buffer ( $20 \mathrm{mM}$ of Tris-base, $192 \mathrm{mM}$ of glycine, $20 \%$ methanol) at $400 \mathrm{~mA}$ for $1.5 \mathrm{~h}$ at room temperature. Membranes were blocked overnight with 5\% (wt/vol) nonfat dry milk in Tris-buffered saline $(10 \mathrm{mM}$ of Tris-base, $150 \mathrm{mM}$ of $\mathrm{NaCl}$ ). For probing for phosphorylated p38 MAPK, the membranes were then incubated for $1 \mathrm{~h}$ with mouse monoclonal anti-human phosphorylated p38 IgM (1:200 dilution; Santa Cruz Biotechnology, Santa Cruz, CA, U.S.A.). Membranes were then washed five times with PBS-Tween $(0.1 \%)$ and incubated for 45 min with anti-mouse IgM-horseradish peroxidase (1:1000 dilution; Santa Cruz Biotechnology). After another five washes, antibody-labeled proteins were detected by Enhanced Chemilumi- nescence as described by the manufacturer (Amersham Pharmacia Biotech, Buckinghamshire, UK). Blots were subsequently stripped (as described by the manufacturer, Santa Cruz Biotechnology) and probed for p38 MAPK using goat polyclonal antihuman p38 MAPK IgG (Santa Cruz Biotechnology).

\section{Statistical Analysis}

All of the experiments in this study were conducted at least three times. The data shown are representative results. Experimental values are expressed as the means \pm SD. The statistical significance of differences between two means was evaluated by $t$ test, and $p \leq 0.05$ was considered significant.

\section{RESULTS}

\section{B. cepacia Induces HLE Cells to Secrete IL-8 in a More Potent Manner than $P$. aeruginosa}

To compare the potency of IL-8 induction between $B$. cepacia and $P$. aeruginosa, we exposed HLE cells to either pathogen at various MOIs ranging between 1:1 and 1000:1 for 4 h. Both B. cepacia and P. aeruginosa induced HLE cells to secrete IL-8 in a MOI-dependent manner after an exposure time of $4 \mathrm{~h}$ (Fig. 1). However, at each MOI tested, IL-8 secreted by $B$. cepacia-infected HLE cells was significantly greater compared with the IL- 8 secreted in response to $P$. aeruginosa over $4 \mathrm{~h}$. Furthermore, significant induction of IL-8 secretion by $B$. cepacia required an MOI of only $>4: 1$ (within 4 h; Fig. 1) compared with $P$. aeruginosa, which was severalfold higher (MOI >64:1).

De novo protein synthesis is required for $I L-8$ secretion by B. cepacia-infected HLE cells. To determine the optimum dose of B. cepacia required to induce IL-8 secretion from HLE cells over $18 \mathrm{~h}$, we infected these cells with increasing MOIs of B. cepacia $(1: 1-500: 1)$ for 18 h. $B$. cepacia was able potently to induce HLE cells to secrete IL-8 in a dose-dependent manner (Fig. 2A). The optimum dose of B. cepacia over $18 \mathrm{~h}$ was at MOI $\geq 40: 1$. Hence, in future experiments, we used an MOI of 50:1 that was near the optimal dose of B. cepacia over an 18-h period. The reason for using an 18-h time point was that as the experiment was continued for $18 \mathrm{~h}$, the IL- 8 signal became much greater, making it easier to quantify the levels of IL-8 secreted by ELISA. The increase in signal for IL-8 secretion also facilitated easier interpretation of inhibition of IL-8 when the inhibitors were used in future studies. It was not possible to incubate A549 cells with intact $P$. aeruginosa for $>4 \mathrm{~h}$ as we found that the viability of the cells was compromised at time points $>4 \mathrm{~h}$ in the presence of this organism. However, B. cepacia did not alter the viability of the cells over $18 \mathrm{~h}$ as indicated in the figure legends.

To investigate whether de novo protein synthesis is required for IL-8 secretion by HLE cells exposed to B. cepacia, we preincubated HLE cells either with the gene transcription inhibitor actinomycin D $(5 \mu \mathrm{g} / \mathrm{mL})$ or with the protein translation inhibitor cycloheximide $(10 \mu \mathrm{g} / \mathrm{mL})$ for $1 \mathrm{~h}$ before exposure to B. cepacia. After $18 \mathrm{~h}$, the culture medium was collected and IL-8 secretion was quantified by ELISA. In the presence of actinomycin D, B. cepacia-induced IL-8 secretion 


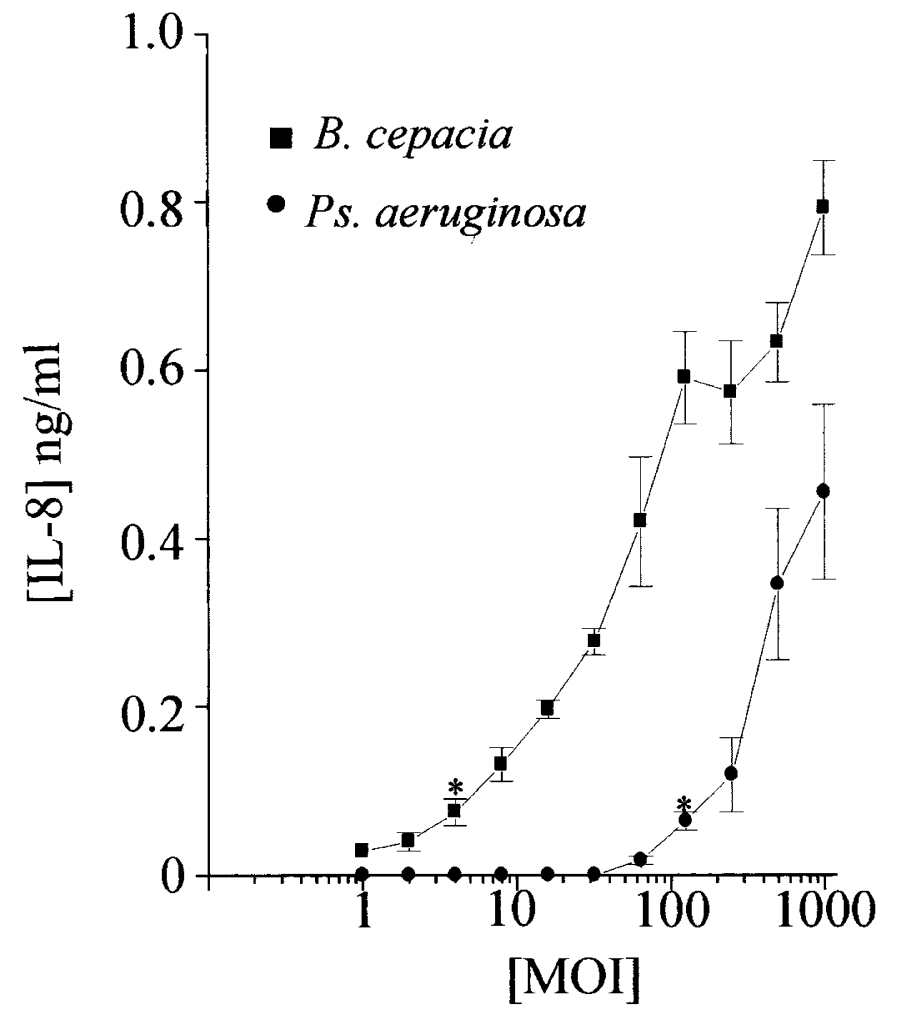

Figure 1. B. cepacia induces HLE cells to secrete IL-8 in a more potent manner than $P$. aeruginosa. HLE cells were infected for $4 \mathrm{~h}$ with increasing MOI (1:1-1000:1) of either B. cepacia $(\square)$ or $P$. aeruginosa $(\mathbf{})$. The culture medium was then removed and centrifuged to remove the bacteria, and ELISAs were performed to detect the level of IL-8. Both B. cepacia and P. aeruginosa induced HLE cells to secrete IL-8 in an MOI-dependent manner after an exposure time of $4 \mathrm{~h}$. However, B. cepacia induced HLE cells to secrete IL- 8 in a more potent manner $(\mathrm{MOI} \geq 4: 1)$ compared with $P$. aeruginosa $(\mathrm{MOI} \geq 64: 1)$. The data are expressed as the mean $\pm \mathrm{SD}$. The results obtained for the test samples were compared with that of cells exposed to media only by the $t$ test with a probability value of $p \leq 0.05$ considered to be significant. $* p \leq 0.05$.

by HLE cells was completely inhibited (Fig. $2 B$ ). Similarly, there was almost complete inhibition of IL-8 secretion by HLE cells exposed to $B$. cepacia in the presence of cycloheximide (Fig. $2 B$ ). These results suggest that $B$. cepacia-induced IL-8 secretion in HLE cells involves de novo IL-8 protein synthesis that occurs at the level of gene transcription.

B. cepacia ATCC strain 700070 induces A549 cells to secrete IL-8 in a similar manner to B. cepacia strain J2315. To elucidate whether $B$. cepacia ATCC strain 700070 induced A549 cells to secrete IL-8 in a similar manner to the highly transmissible strain of $B$. cepacia strain $\mathrm{J} 2315$, we incubated HLE cells with either strain for $18 \mathrm{~h}$ and then tested for IL-8 secretion as previously described. We found that both strains of B. cepacia induced HLE cells to secrete IL- 8 in a similar manner (Fig. 3). Both of these strains belong to genomovar III of the B. cepacia complex.

\section{B. cepacia-Induced IL-8 Secretion by HLE Cells Is CD14 Dependent and Is Not Down-Regulated Posttreatment with PK}

To elucidate whether IL-8 secretion by $B$. cepacia-infected HLE cells was being mediated by a protein component on the
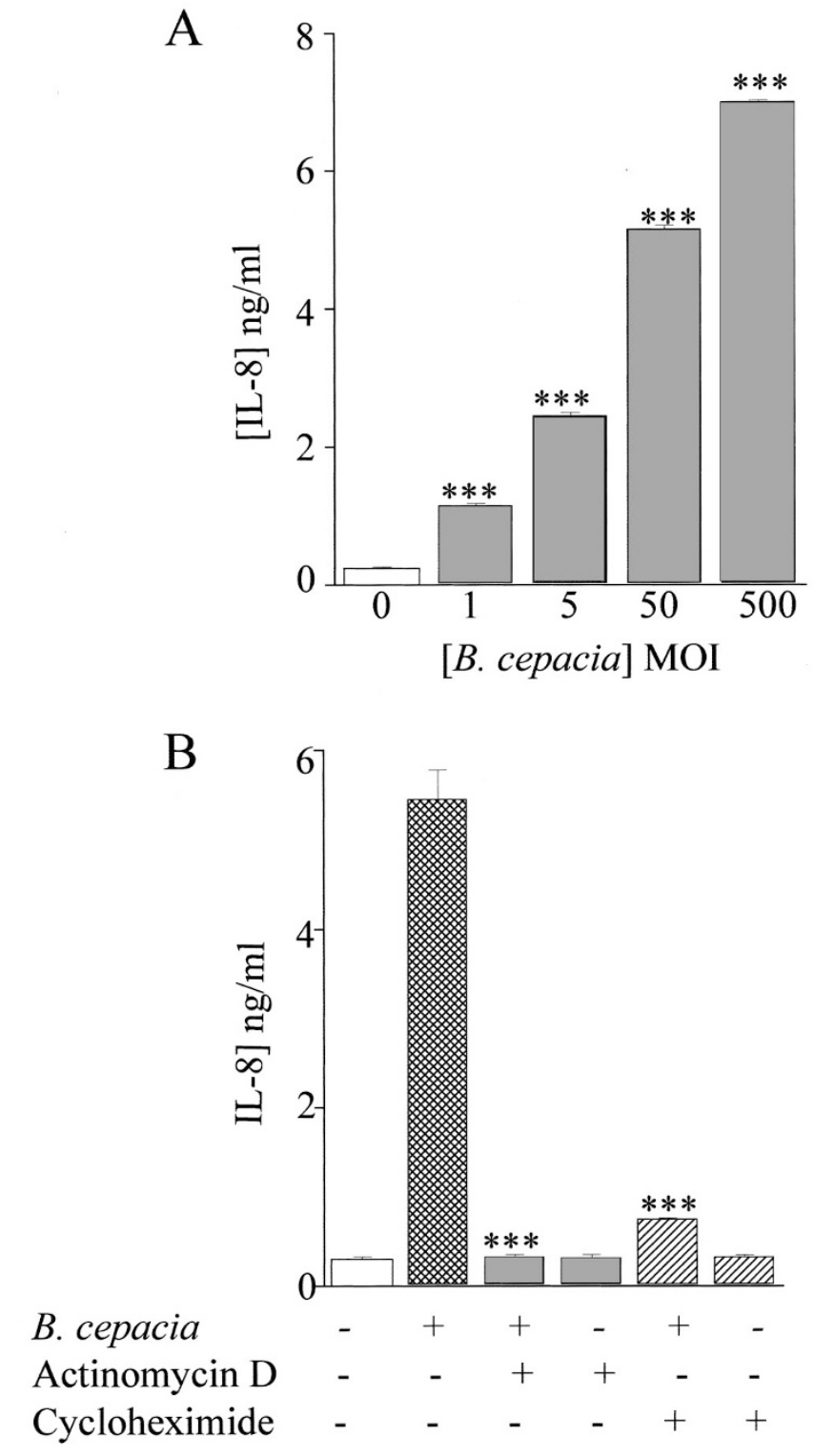

Figure 2. B. cepacia-induced IL-8 secretion is MOI dependent and requires de novo protein synthesis. HLE cells were infected with B. cepacia (MOI 1:1-500:1) for $18 \mathrm{~h}$. The culture medium was then removed and centrifuged to remove the bacteria, and ELISAs were performed to detect the level of IL-8. HLE cells exposed to $B$. cepacia significantly released nanogram levels of IL-8 at an MOI from as low as 1:1 up to 500:1 (圈) compared with cells that received media alone ( $\square$ ). The optimum dose of B. cepacia-induced IL-8 secretion by HLE cells over $18 \mathrm{~h}$ was 40:1 $(A)$. HLE cells were preexposed to either actinomycin D $(5 \mu \mathrm{g} / \mathrm{mL})$ or cycloheximide $(10 \mu \mathrm{g} / \mathrm{mL}) 1 \mathrm{~h}$ before infecting with $B$. cepacia (MOI 50:1). The culture media was then removed, and IL-8 was quantified by ELISA. Actinomycin D $(5 \mu \mathrm{g} / \mathrm{mL})$ or cycloheximide $(10$ $\mu \mathrm{g} / \mathrm{mL}$ ) alone failed to induce IL-8 synthesis. Actinomycin D (圈) and cycloheximide (שC) inhibited B. cepacia-induced IL-8 synthesis by $100 \%$ and $80 \%$, respectively $(B)$. The data are expressed as the mean $\pm \mathrm{SD}$. The data obtained for the test samples were compared with that of cells exposed to B. cepacia in the absence of inhibitors (柯) by the $t$ test with a probability value of $p \leq 0.05$ considered to be significant. $* * * p<0.001$.

bacteria, we digested B. cepacia with $\mathrm{PK}$ as described in "Methods." After treating B. cepacia with $0.1 \mathrm{mg} / \mathrm{mL}$ of PK, there was total bacterial protein digestion as monitored by SDS-PAGE (data not shown). There was no significant difference in IL-8 secretion by HLE cells infected with either 




Figure 3. B. cepacia strain ATCC 700070 induces HLE cells to secrete IL-8 in a similar manner to the highly transmissible strain of $B$. cepacia strain J2315. HLE cells were infected with either B. cepacia strain ATCC 700070 or $B$. cepacia $\mathrm{J} 2315$ for $18 \mathrm{~h}$. The culture medium was then removed and centrifuged to remove the bacteria, and ELISAs were performed to detect the level of IL-8 secreted. Both strains of B. cepacia induced HLE cells to secrete IL-8 in a dose-dependent and similar manner. B. cepacia strain ATCC 700070 was confirmed to belong to genomovar III of the B. cepacia complex.

PK-treated B. cepacia compared with untreated B. cepacia (Fig. 4A). These data suggest that the component of $B$. cepacia responsible for IL-8 secretion by HLE cells was not likely to be a protein. To determine whether LPS was the critical component necessary for IL-8 synthesis, we investigated the role of the LPS receptor CD14 as described in "Methods." In the presence of anti-CD14 ( $\alpha$ CD14, MY4) receptor antibody, IL-8 secretion by B. cepacia was significantly abrogated (Fig. $4 B$ ), thus suggesting that B. cepacia-induced IL-8 synthesis in HLE cells is CD14 dependent. IL-8 synthesis by HLE cells exposed to the positive control $P$. aeruginosa LPS (serotype 10) was completely abrogated in the presence of $\alpha \mathrm{CD} 14$ receptor antibody (Fig. 4B). This abrogation was not due to nonspecific binding by the antibody as the isotypic control antibody failed to inhibit IL-8 secretion by either $B$. cepacia or $P$. aeruginosa LPS. As capturing of LPS by CD14 receptors is facilitated by a serum component known as LPS-binding protein (LBP), we tested whether increasing the percentage of serum $(0-2 \%)$ increased B. cepacia-induced IL-8 synthesis. We found that IL-8 secretion by $B$. cepacia-infected HLE cells was serum sensitive. As the percentage of serum was increased from 0 to $2 \%$, IL-8 secretion by HLE cells infected by $B$. cepacia also increased (data not shown). This provides further evidence to support the observation that $B$. cepacia-LPS may be the component responsible for IL-8 induction in HLE cells. In addition, polymyxin-B inhibited B. cepacia-induced IL-8 secretion



Figure 4. The IL-8-inducing component of B. cepacia is not a protein and is CD14 dependent. B. cepacia was treated with PK $(0.1 \mathrm{mg} / \mathrm{mL})$ and incubated at $37^{\circ} \mathrm{C}$ for $1 \mathrm{~h}$. PMSF was then added to stop the reaction. HLE cells were infected with PK-treated B. cepacia (MOI 50:1), and the IL-8 released by HLE



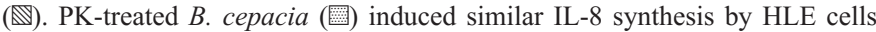
compared with untreated B. cepacia (网), suggesting that the induction of IL-8 is not due to a protein $(A)$. For testing whether IL-8 synthesis was CD14 dependent, HLE cells were preincubated with $\alpha \mathrm{CD} 14$ receptor antibody for $1 \mathrm{~h}$ before treating with either B. cepacia or P. aeruginosa (serotype 10) LPS. After $18 \mathrm{~h}$, the culture medium was removed and tested for IL- 8 synthesis by ELISA. The isotypic control $\operatorname{IgG} 2 \mathrm{~b}$ was also used to test for nonspecific binding (相). In the presence of $\alpha \mathrm{CD} 14$ receptor antibody, B. cepacia-induced IL-8 secretion was significantly abrogated ( $B$, 娄). Anti-CD14 receptor antibody also abrogated $P$. aeruginos $a$ LPS-induced IL- 8 secretion $(\mathbb{Q})$. The data are expressed as the mean $\pm \mathrm{SD}$. The results obtained for the test samples were compared with that of cells exposed to untreated B. cepacia (网) or $P$. aeruginosa LPS $(\mathbb{\mathbb { N }})$ by the $t$ test with a probability value of $p \leq 0.05$ considered to be significant. ${ }^{* * *} p<0.001$. 
(data not shown), providing further confirmation that IL-8 secretion by $B$. cepacia-infected alveolar epithelial cells is via the LPS of this microorganism.

\section{IL-8 Secretion in B. cepacia-Infected HLE Cells Does Not Require IL-1 $\beta$ Synthesis}

For determining whether HLE cells expressed an increase in mRNA for IL- $1 \beta$ or IL-8 upon exposure to $B$. cepacia, HLE cells were infected with $B$. cepacia and incubated for $18 \mathrm{~h}$ at $37^{\circ} \mathrm{C}$ before extraction of the RNA. The mRNA was isolated, and RT-PCR for IL-8 and IL- $1 \beta$ was performed as described in "Methods." B. cepacia-infected HLE cells displayed a significant (1.3-fold) increase in IL-8 mRNA compared with basal levels (Fig. 5) as quantified by densitometry. However, in contrast, mRNA expression for IL- $1 \beta$ in HLE cells did not significantly change after exposure to $B$. cepacia (Fig. 5). We also failed to detect any IL-1 $\beta$ secreted into the culture medium by HLE cells $18 \mathrm{~h}$ after exposure to $B$. cepacia by ELISA. In contrast, HLE cells infected by $B$. cepacia in the same manner secreted at least $5 \mathrm{ng} / \mathrm{mL}$ of IL- 8 (Fig. $2 A$ ). These data suggest that IL- 8 gene transcription by HLE cells in the presence of $B$. cepacia may be occurring independent of IL- $1 \beta$ synthesis.

Inhibition of $p 38$ and ERK MAPK activation downregulates $I L-8$ secretion by B. cepacia-induced HLE cells. The signaling proteins activated by HLE cells to secrete IL-8 by $B$. cepacia are unknown. In general, as the MAPK signaling pathways have been reported to be involved in IL-8 secretion, we preincubated HLE cells with either the p38 MAPK inhibitor SB203580 or the ERK MAPK inhibitor PD98059 as described in "Methods." Pretreatment with SB203580 $(3 \mu \mathrm{M})$ inhibited $\geq 80 \%$ of the IL-8 secretion in HLE cells infected with $B$. cepacia (Fig. $6 A$ ). In contrast, IL-8 secretion in the presence of the ERK MAPK inhibitor PD98059 $(10 \mu \mathrm{M})$ was abrogated by only $\geq 30 \%$ (Fig. $6 A$ ). Western blot analysis was performed to confirm whether resting levels of p38 MAPK were phosphorylated postinfection by B. cepacia. There was a marked increase in phosphorylated p38 protein after exposing HLE cells to $B$. cepacia for $10 \mathrm{~min}$ (Fig. $6 \mathrm{~B}$ ). This phosphorylation for p38 protein was inhibited in the presence of the p38 MAPK inhibitor SB203580, confirming that the p38 MAPK pathway is an integral pathway during $B$. cepacia-induced IL-8 synthesis in HLE cells. There was little or no difference in the basal levels of phosphorylated $\mathrm{p} 38$ protein in cells exposed to media and SB203580 only (data not shown).

To test whether IL-8 secretion in B. cepacia-infected HLE cells was mediated via prostaglandin or protein kinase $C$ (PKC), we preincubated B. cepacia with the prostaglandin inhibitor indomethacin $(50 \mu \mathrm{M}$; Calbiochem $)$ or the PKC inhibitor GF109203X (5 $\mu \mathrm{M}$; Calbiochem). There was no inhibition of IL-8 secretion by B. cepacia-infected HLE cells in the presence of either indomethacin or GF109203X. HLE cells treated with either indomethacin $(50 \mu \mathrm{M})$ or GF109203X $(5 \mu \mathrm{M})$ alone failed to induce IL-8 secretion.



Figure 5. Induction of only IL- 8 mRNA and not IL-1 $\beta$ occurs in HLE cells after exposure to $B$. cepacia. HLE cells were infected with $B$. cepacia (MOI 50:1) for $18 \mathrm{~h}$. The culture medium was then removed, and RT-PCR was performed on the cells to determine increases in mRNA expression for IL-8 or IL- $1 \beta$. HLE cells showed significant induction of mRNA levels only for IL-8 but not for IL- $1 \beta$ after exposure to $B$. cepacia, compared with cells in the absence of $B$. cepacia. The data are expressed as densitometry units of each cytokine band obtained by RT-PCR. B. cepacia-infected cytokine levels were compared with basal levels (control, media alone, $\square$; infected by B. cepacia, 圈). $\beta$-Actin was used as a loading control. The data obtained for the test samples were compared with that of cells exposed to media only by the $t$ test with a probability value of $p \leq 0.05$ considered to be significant. $* * * p<$ 0.001 .

\section{Inhibition of NF-kB Down-Regulates IL-8 Secretion by B. cepacia-Induced HLE Cells}

For investigating whether NF- $\kappa \mathrm{B}$ was involved during $B$. cepacia-induced IL-8 secretion, HLE cells were exposed to the general $\mathrm{I} \kappa \mathrm{B}$ inhibitor dexamethasone $(1-1000 \mathrm{nM})(20,21)$ for $1 \mathrm{~h}$ before infection with B. cepacia. After $18 \mathrm{~h}$ of incubation, the IL- 8 secreted into the culture medium was quantified by ELISA. Dexamethasone at a concentration as low as $10 \mathrm{nM}$ was able to inhibit significantly IL-8 secretion by HLE cells exposed to B. cepacia (Fig. 7). Furthermore, this inhibition of IL-8 secretion was found to be dose dependent, where $1 \mu \mathrm{M}$ of dexamethasone was able to inhibit IL-8 secretion by $>80 \%$.

\section{DISCUSSION}

Proinflammatory cytokines have been implicated as playing an essential role in the pathogenesis of CF infection $(5,7)$, and pathogens such as $B$. cepacia are significantly responsible for this cytokine induction. As B. cepacia is a multidrug-resistant microorganism, treatment of patients who have CF and are infected with this bacteria is increasingly difficult $(22,23)$. 


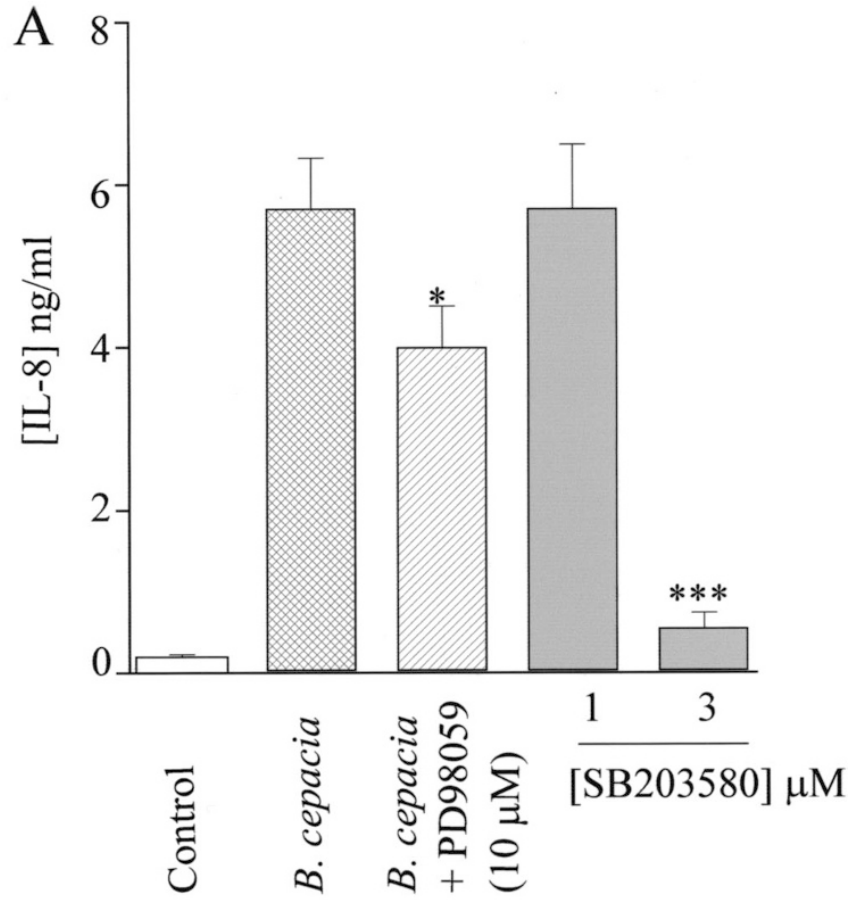

B

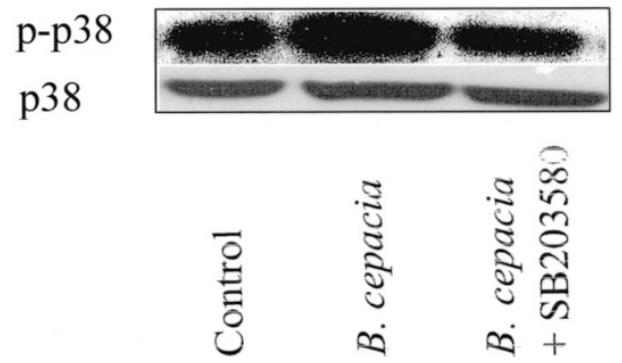

Figure 6. B. cepacia induces phosphorylation of p38 MAPK in HLE cells. HLE cells were preincubated with the p38 MAPK inhibitor SB203580 (1-3 $\mu \mathrm{M}$ ), $1 \mathrm{~h}$ before infecting with $B$. cepacia (MOI 50:1). The conditioned medium was then collected, and IL-8 release was quantified by ELISA. Western blot analysis was also performed to detect the phosphorylation of p38 MAPK protein. In the presence of $10 \mu \mathrm{M}$ of PD98509, 30\% of IL-8 secretion was abrogated by $B$. cepacia-induced HLE cells (ש) in comparison with $B$. cepacia in the absence of PD98509 (四). A total of 3-10 $\mu \mathrm{M}$ of SB203580 inhibited B. cepacia-induced IL-8 synthesis by $80 \%$ (畻) compared with $B$. cepacia in the absence of SB203580 (A, 四). SB203580 or PD98509 alone failed to induce HLE cells to secrete IL-8. A marked increase in the phosphorylated p38 MAPK protein occurred within 10 min of infecting HLE cells with $B$. cepacia as monitored by Western analysis. This phosphorylation of p38 MAPK protein was then inhibited down to basal levels in the presence of $3 \mu \mathrm{M}$ of SB203580 $(B)$. The data are expressed as the mean $\pm \mathrm{SD}$. The results obtained for the test samples were compared with that of cells exposed to $B$. cepacia in the absence of inhibitors by the $t$ test with a probability value of $p$ $\leq 0.05$ considered to be significant. ${ }^{*} p<0.05,{ }^{* * *} p<0.001$.

Hence, further information on the signaling pathways triggered by $B$. cepacia to induce cytokines is particularly valuable to design potential therapeutics that may decrease the overexpression of cytokines.

In this study, we determined that B. cepacia at an MOI $>4: 1$ significantly induced HLE cells to secrete IL-8; in contrast, $P$. aeruginosa required an $\mathrm{MOI}>64: 1$ for significant induction of

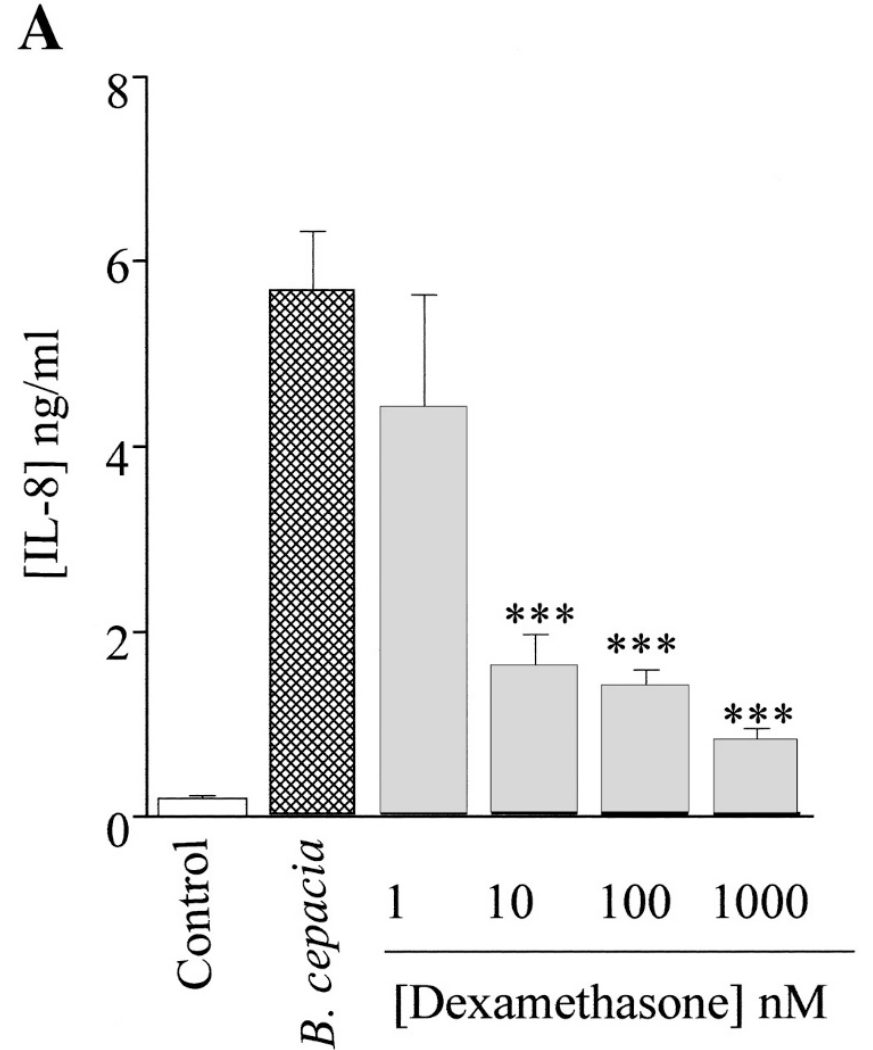

Figure 7. Inhibition of NF- $\kappa \mathrm{B}$ down-regulates IL-8 secretion by B. cepaciainduced HLE cells. HLE cells were exposed to 1-1000 nM of dexamethasone $1 \mathrm{~h}$ before infecting with $B$. cepacia. The culture medium was then collected, and secreted IL- 8 was quantified by ELISA. A total of $10 \mathrm{nM}$ of dexamethasone significantly inhibited $B$. cepacia-induced IL- 8 synthesis by HLE cells by $\geq 60 \%$ (畻) in comparison with untreated $B$. cepacia (四). This inhibition was dose dependent, and maximal inhibition was observed at $1 \mu \mathrm{M}$ of dexamethasone. HLE cells exposed to dexamethasone alone failed to secrete IL-8. The results obtained for the test samples were compared with that of cells exposed to $B$. cepacia in the absence of dexamethasone by the $t$ test with a probability value of $p \leq 0.05$ considered to be significant. ${ }^{* *} p<0.001$.

IL-8 secretion to occur. This finding is consistent to a recent study by Zughaier et al. (24), who demonstrated that the LPS from B. cepacia (strain J2315) is 10-fold more active in the induction of TNF- $\alpha$ by human monocytes compared with the LPS from $P$. aeruginosa (strain PAO1). However, as we did not achieve a maximal dose for $P$. aeruginosa, we cannot draw any conclusions regarding the efficacy of $P$. aeruginosa compared with B. cepacia.

Of the species of Burkholderia, those from genomovar III account for most of the clinical isolates and most of the epidemic strains. We compared the ability of two isolates from genomovar III (ET12, strain J2315 and ATCC 700070) to induce HLE cells to secrete IL-8. Both strains of B. cepacia were equally potent in the induction of IL- 8 by HLE cells. Furthermore, this is the first report that confirms that $B$. cepacia ATCC strain 700070 belongs to genomovar III of the $B$. cepacia complex (J. Lipuma, personal communication).

In this study, we found that in the presence of actinomycin D (gene transcription inhibitor), B. cepacia-induced IL-8 secretion by HLE cells was completely inhibited. Similarly, there was $\geq 80 \%$ inhibition of IL-8 secretion by HLE cells exposed 
to $B$. cepacia in the presence of cycloheximide (protein translation inhibitor), suggesting that $B$. cepacia-induced IL-8 secretion in HLE cells involves de novo IL-8 protein synthesis and gene transcription.

PK-treated B. cepacia was able to induce IL- 8 synthesis by HLE cells in a comparable manner to untreated $B$. cepacia, thus suggesting that the IL- 8 -inducing component of $B$. cepacia is not a protein but may be due to LPS. Further evidence to support this was obtained by the use of $\alpha \mathrm{CD} 14$ receptor antibody. B. cepacia-induced IL-8 release by HLE cells was significantly abrogated by the use of $\alpha \mathrm{CD} 14$ receptor antibody, indicating that IL- 8 secretion is CD14 dependent. CD14 receptors are specific for LPS, their main role being in capturing LPS and then presenting the LPS to other receptors such as Toll-like receptors. The capturing of LPS by CD14 receptors is facilitated by a serum protein, LBP. In our studies, we found that in the presence of serum there was a dose-dependent increase in the IL-8 induction in B. cepacia-infected HLE cells (data not shown). The IL-8-inducing capability of $B$. cepacia was dependent on the percentage of serum present, suggesting 1) that as the serum concentration was increased, there may be more LBP present to facilitate LPS binding to CD14 receptors, and 2) that there also may be soluble CD14 (sCD14) receptors present in the serum. Therefore, as the percentage of serum was increased, the concentration of $\mathrm{sCD} 14$ receptors may also increase, allowing more LPS to bind. Our finding is consistent with a recent study by Zughaier et al. (24), who demonstrated that the LPS from other clinical isolates of $B$. cepacia induced TNF- $\alpha$ in human monocytes in a CD14-dependent manner.

In contrast, a study by Palfreyman et al. (14) reported that a non-LPS extracellular factor from $B$. cepacia induced IL-8 secretion in alveolar epithelial cells. The differences between our data and their data may be due to differences in the $B$. cepacia strains used between the two studies and also to the use of whole organisms (in this study) and only the culture medium (in their study).

We found no significant change in the expression of IL-1 $\beta$ mRNA or in the secretion of IL- $\beta$ in HLE cells infected with B. cepacia, indicating that perhaps IL-8 induction by HLE cells exposed to $B$. cepacia occurs via an IL- $1 \beta$-independent manner. This is at present under investigation in our laboratory. A recent report to support this observation demonstrated that the LPS from an environmental strain of B. cepacia (ATCC 25416) stimulated murine macrophages to secrete IL-6 and TNF with relative lack of IL- $1 \beta$-inducing ability (25). A similar trend has been observed with a periodontal pathogen, Actinobacillus actinomycetemcomitans, implicated in juvenile periodontitis, where synthesis of IL-6 secretion in gingival fibroblasts occurs independent of IL-1 $\beta$ synthesis (26).

B. cepacia-induced IL-8 secretion in HLE cells may occur via the activation of the p38 MAPK pathway as significant inhibition of IL- 8 was found after co-incubating $B$. cepacia with the p38 MAPK inhibitor SB203580. There was a marked increase in the protein for phosphorylated p38 MAPK after exposing HLE cells with B. cepacia. This enhancement for phosphorylated p38 MAPK protein was significantly diminished in the presence of the p38 MAPK inhibitor SB203580. In fact, SB203580 at a concentration as low as $3 \mu \mathrm{M}$ was able to diminish IL-8 secretion by $\geq 80 \%$. The ERK MAPK pathway may also play a role in $B$. cepacia-induced IL-8 secretion in HLE cells, as a specific inhibitor of ERK MAPK PD98059 $(10 \mu \mathrm{M})$ inhibited approximately $30 \%$ of IL- 8 synthesis induced by $B$. cepacia. The role of the JNKs was not pursued in this study as there are no specific inhibitors for these kinases. Furthermore, as an SB203580 concentration as low as $3 \mu \mathrm{M}$ was able to reduce IL- 8 secretion by $>80 \%$ in HLE cells exposed to $B$. cepacia, we speculate that the JNK pathway by itself would be insufficient for expression of IL-8 genes postinfection with $B$. cepacia in HLE cells. Our observation correlates to a recent report that demonstrates that both the p38 and ERK MAPKs are activated after exposure to pyocyanin from $P$. aeruginosa by human airway epithelial cells to induce IL-8 secretion (27). In addition, they show that this IL-8 secretion is independent of PKC. In our study, we also found that B. cepaciainduced IL-8 secretion by HLE cells was not abrogated in the presence of inhibitors for PKC. Ratner et al. (28) also demonstrated that $P$. aeruginosa and Staphylococcus aureus activated both p38 and ERK MAPK pathways in $1 \mathrm{HAEo}^{-}$tracheal cells. This activation occurred within $30 \mathrm{~min}$ of exposure to $P$. aeruginosa and $60 \mathrm{~min}$ of exposure to $S$. aureus. In this study, phosphorylation of p38 occurred within $10 \mathrm{~min}$ of exposure to $B$. cepacia.

It has long been known that dexamethasone induces transcription of the gene for the inhibitory protein $\mathrm{I} \kappa \mathrm{B} \alpha$, an inhibitor of the translocation of $\mathrm{NF}-\kappa \mathrm{B}$, one of the transcriptional control elements for the IL- 8 gene $(20,21)$. NF- $\kappa \mathrm{B}$ is normally retained in the cytoplasm through its association with the inhibitory molecule $\mathrm{I} \kappa \mathrm{B}$. Phosphorylation, ubiquination, and proteolysis of $\mathrm{I} \kappa \mathrm{B}$ allows $\mathrm{NF}-\kappa \mathrm{B}$ to translocate to the nucleus and induces transcription of cytokine gene expression (29). In the presence of dexamethasone (10-1000 nM), IL-8 secretion by $B$. cepacia-infected HLE cells was significantly abrogated. The finding that $B$. cepacia-induced IL-8 synthesis by HLE cells is blocked by dexamethasone strongly suggests that NF- $\kappa \mathrm{B}$ is a key transcriptional element involved in IL-8 synthesis by this stimulus. The involvement of NF- $\kappa \mathrm{B}$ in our study strongly corresponds to a recent study that showed that there were elevated levels of NF- $\kappa \mathrm{B}$ in $B$. cepacia-infected Cftr $-/-$ mice (30). Clinically, the down-regulation of NF- $\kappa \mathrm{B}$ may be therapeutically beneficial as a recent report by Kazachkov et al. (31) described that a patient who had CF and was infected by $B$. cepacia and had extreme respiratory failure survived her illness after treatment with corticosteroids. They speculated that treatment with the corticosteroids might have decreased the $B$. cepacia-induced influx of proinflammatory cytokines and neutrophil-induced inflammation.

We conclude that a CF clinical isolate of $B$. cepacia (ATCC 700070) potently induces IL-8 synthesis in HLE cells in a CD14- and MAPK-dependent manner. Colonization of B. cepacia in patients with CF may lead to extensive activation of the MAPKs, thus inducing overexpression of IL-8, consequently leading to tissue damage as a result of an increase in neutrophil activity as observed in patients with $\mathrm{CF}$. The data from this study underscore the importance of defining the signaling pathways activated after exposure to $B$. cepacia to facilitate the design of future therapies to minimize acute inflammation in CF. 


\section{REFERENCES}

1. Bye MR, Ewig JM, Quitell LM 1994 Cystic fibrosis. Lung 172:251-270

2. Auerbach HS, Williams M, Kirkpatrick J, Cohen HR 1985 Alternate-day prednisone reduces morbidity and improves pulmonary function in cystic fibrosis. Lancet 2:686-688

3. Govan JRW, Hughes JE, Vandamme P 1996 Burkholderia cepacia: medical, taxonomic and ecological issues. J Med Microbiol 45:395-407

4. Isles A, Maclusky I, Corey M, Gold R, Prober C, Fleming P, Levison H 1984 Pseudomonas cepacia infection in cystic fibrosis: an emerging problem. J Pediatr 104:206-210

5. Bonfield TL, Panuska JR, Konstan MW, Hilliard KA, Hilliard JB, Ghnaim H, Berger M 1995 Inflammatory cytokines in cystic fibrosis lungs. Am J Respir Crit Care Med $152: 2111-2118$

6. Norman D, Elborn JS, Cordon SM, Rayner RJ, Wiseman MS, Hiller EJ, Shale DJ 1991 Plasma tumour necrosis factor alpha in cystic fibrosis. Thorax 46:91-95

7. Dean TP, Dai Y, Shute JK, Church MK, Warner JO 1993 Interleukin-8 concentrations are elevated in bronchoalveolar lavage, sputum, and sera of children with cystic fibrosis. Pediatr Res 34:159-161

8. Keane MP, Strieter RM 2000 Chemokine signaling in inflammation. Crit Care Med 28:N13-N26

9. Nick JA, Avdi NJ, Young SK, Lehman LA, McDonald PP, Frasch SC, Billstrom MA, Henson PM, Johnson GL, Wrthen G 1999 Selective activation and functional significance of p38 alpha mitogen-activated protein kinase in lipopolysaccharidestimulated neutrophils. J Clin Invest 103:851-858

10. Burns JL, Jonas Mechthild J, Chi EY, Clark DK, Berger A, Griffith A 1996 Invasion of respiratory epithelial cells by Burkholderia (Pseudomonas) cepacia. Infect Immun 64:4054-4059

11. Martin DW, Mohr CD 2000 Invasion and intracellular survival of Burkholderia cepacia Infect Immun 68:24-29

12. Keig PM, Ingham E, Kerr KG 2001 Invasion of human type II pneumocytes by Burkholderia cepacia. Microb Pathog 30:167-170

13. Mori N, Oishi K, Sar B, Mukaida N, Nagatake T, Matsushima K, Yamamoto N 1999 Essential role of transcription factor nuclear factor- $\kappa \mathrm{B}$ in regulation of interleukin- 8 gene expression by nitrite reductase from Pseudomonas aeruginosa in respiratory epithelial cells. Infect Immun 67:3872-3878

14. Palfreyman RW, Watson ML, Eden C, Smith AW 1997 Induction of biologically active interleukin-8 from lung epithelial cells by Burkholderia (Pseudomonas) cepacia products. Infect Immun 65:617-622

15. Leidal KG, Munson KL, Denning GM 2001 Small molecular weight secretory factors from Pseudomonas aeruginosa have opposite effects on IL-8 and Rantes expression by human airway epithelial cells. Am J Respir Cell Mol Biol 25:186-195

16. Burns JL, Wadsworth CH, Barry J, Goodall CP 1996 Nucleotide sequence analysis of a gene from Burkholderia (Pseudomonas) cepacia encoding an outer membrane lipoprotein involved in multiple antibiotic resistance. Antimicrob Agents Chemother 40:307-313

17. Steffen MJ, Ebersole JL 1996 Sequential ELISA for cytokine levels in limited volumes of biological fluids. Biotechniques 21:504-509
18. Weinberg A, Holt SC 1990 Interaction of Treponema denticola TD-4, GM-1, and MS25 with human gingival fibroblasts. Infect Immun 58:1720-1729

19. Jung CJ, Eckmann L, Yang SK, Panja A, Fierer J. Morzycka-Wroblewsa E, Kagnoff MF 1995 A distinct array of pro-inflammatory cytokines is expressed in human colon epithelial cells in response to bacterial invasion. J Clin Invest 95:55-65

20. Auphan N, Didonato JA, Rosette C, Helmberg A, Karin M 1995 Immunosuppression by glucocorticoids: inhibition of NF- $\kappa \mathrm{B}$ activity through induction of $\mathrm{I} \kappa \mathrm{B}$ synthesis. Science 270:283-286

21. De Vera ME, Taylor BS, Wang Q, Shapiro RA, Billiar TR, Geller DA 1997 Dexamethasone suppresses iNOS gene expression by upregulating I-kappa B alpha and inhibiting NF-kappa B. Am J Physiol 273:G1290-G1296

22. Lewin C, Doherty C, Govan JRW 1993 In vitro activities of meropenem, PD 127391, PD 131628, ceftazidime, chloroamphenicol, co-trimoxazole, and ciprofloxacin against Pseudomonas cepacia. Antimicrob Agents Chemother 37:123-125

23. Simpson IN, Finlay J, Winstanley DJ, Dewhurst N, Nelson J, Butler S, Govan JRW 1994 Multi-resistance isolates possessing characteristics of both Burkholderia (Pseudomonas) cepacia and Burkholderia gladioli from patients with cystic fibrosis. J Antimicrob Chemother 34:353-361

24. Zughaier SM, Ryley HC, Jackson SK 1999 Lipopolysaccharide (LPS) from Burkholderia cepacia is more active than LPS from Pseudomonas aeruginosa and Stenotrophomonas maltophilia in stimulating tumor necrosis factor alpha from human monocytes. Infect Immun 67:1505-1507

25. Shimomura H, Matsuuraa M, Saito S, Hirai Y, Isshiki Y, Kawahara K 2001 Lipopolysaccharide of Burkholderia cepacia and its unique character to stimulate murine macrophages with relative lack of interleukin- $1 \beta$-inducing ability. Infect Immun 69:3663-3669

26. Reddi K, Nair SP, White PA, Hodges S, Tabona P, Meghji S, Poole S, Wilson M, Henderson B 1996 Surface-associated material from the bacterium Actinobacillus actinomycetemcomitans contains a peptide which, in contrast to lipopolysaccharide, directly stimulates fibroblast interleukin-6 gene transcription. Eur J Biochem 236 871-876

27. Denning GM, Wollenweber LA, Railsback MA, Cox CD, Stoll LL, Britigan BE 1998 Pseudomonas pyocyanin increases interleukin- 8 expression by human airway epithelial cells. Infect Immun 66:5777-5784

28. Ratner AJ, Bryan R, Weber A, Nguyen S, Barnes D, Pitt A, Gelber S, Cheung A, Prince A 2001 Cystic fibrosis pathogens activate Ca2+-dependent mitogen-activated protein kinase signaling pathways in airway epithelial cells. J Biol Chem 276:1926719275

29. Abraham E 2000 NF- $\kappa$ B activation. Crit Care Med 28:N100-N104

30. Sajjan U, Thanassoulis G, Cherapanov V, Lu A, Sjolin C, Steer B, Wu YJ, Rotstein OD, Kent G, Mckerlie C, Forstner J, Downey GP 2001 Enhanced susceptibility to pulmonary infection with Burkholderia cepacia in Cftr-/- mice. Infect Immun 69:5138-5150

31. Kazachkov M, Lager J, Lipuma J, Barker PM 2001 Survival following Burkholderia cepacia sepsis in a patient with cystic fibrosis treated with corticosteroids. Pediat Pulmonol 32:338-340 